\title{
Clinical Characteristics and Prognosis of Allergic Bronchopulmonary Aspergillosis: A Retrospective Cohort Study
}

\author{
Yingying Zeng ${ }^{1,2}$ \\ Xiaomin Xue ${ }^{1,2}$ \\ Hui Cai ${ }^{1,2}$ \\ Guiping Zhu ${ }^{1,2}$ \\ Mengchan Zhu ${ }^{1,2}$ \\ Jian Wang ${ }^{1,2}$ \\ Xixi Song ${ }^{1,2}$ \\ Yuqing Mo $\mathrm{M}^{\mathrm{I,2}}$ \\ Xin Gao ${ }^{1,2}$ \\ Jiaye Zhou $^{3}$ \\ Ling Ye iD ${ }^{1,2}$ \\ Meiling Jin ${ }^{1,2}$ \\ 'Department of Respiratory and Critical \\ Care Medicine, Zhongshan Hospital, \\ Fudan University, Shanghai, People's \\ Republic of China; ${ }^{2}$ Department of \\ Allergy, Zhongshan Hospital, Fudan \\ University, Shanghai, People's Republic of \\ China; ${ }^{3}$ Department of Laboratory \\ Medicine, Zhongshan Hospital, Fudan \\ University, Shanghai, People's Republic of \\ China
}

Correspondence: Meiling Jin; Ling Ye Department of Respiratory and Critical Care Medicine, Zhongshan Hospital, Fudan University, 180 Fenglin Road, Shanghai, 200032, People's Republic of China

Tel +862I-64041990

Email mljinII8@I63.com;

ye.ling@zs-hospital.sh.cn
Background: The clinical features, treatment, and prognosis of allergic bronchopulmonary aspergillosis (ABPA) are not well-defined.

Objective: We aimed to investigate the clinical characteristics, therapy, and prognosis of ABPA to aid its clinical recognition.

Methods: A total of 232 patients with ABPA were analyzed retrospectively. The characteristics of ABPA in terms of its misdiagnosis, computed tomography classification, therapy, and its relationship with asthma were analyzed, and risk factors for acute exacerbation of ABPA were analyzed based on follow-up data.

Results: Of the 232 ABPA patients, 132 had a history of misdiagnosis. Compared with the misdiagnosed patients, ABPA patients with central bronchiectasis, a high total eosinophil count, and mucus plugs were less likely to be misdiagnosed. Compared with serological ABPA, ABPA with central bronchiectasis was more likely to occur in older people and in patients with mucus plugs, and decreased forced vital capacity and diffusing capacity for carbon monoxide. ABPA patients with asthma were more likely to have bronchiectasis, decreased lung function in $1 \mathrm{~s}$ FEV1 and FEV1/FVC, and shorter time to first acute exacerbation compared with ABPA patients without asthma. Patients receiving glucocorticoids plus antifungal therapy had a longer time to first exacerbation than those receiving glucocorticoid therapy alone. Univariate and multivariate analyses revealed that duration of asthma history, duration of misdiagnosis, mucus plugs, and poor pulmonary function were risk factors for acute exacerbation of ABPA.

Conclusion: To our knowledge, this is the largest sample size study of ABPA in China. ABPA patients with a history of asthma and/or central bronchiectasis on high-resolution computed tomography are prone to frequent acute exacerbations. The use of glucocorticoids combined with antifungal drugs can prolong the time to the first acute exacerbation in ABPA patients. Longer durations of asthma history and misdiagnosis, mucus plugs, and poor pulmonary function are risk factors for acute exacerbation of ABPA.

Keywords: allergic bronchopulmonary aspergillosis, central bronchiectasis, diagnosis, follow-up, treatment

\section{Introduction}

Allergic bronchopulmonary aspergillosis (ABPA) is a complex pulmonary disorder resulting from immune hypersensitivity to airway colonization by Aspergillus fumigatus. $^{1,2}$ The diagnosis of ABPA is based on a combination of clinical, biological and radiological manifestations and diagnosis criteria. ${ }^{3,4}$ However, despite these diagnostic criteria, the diagnosis of ABPA remains difficult. 
ABPA is currently classified into five stages by the International Society for Human and Animal Mycology (ISHAM) Working Group. ${ }^{3}$ Previous studies have shown that patients with stage I-IV ABPA can enter complete remission, but patients with stage $\mathrm{V}$ are more likely to develop pulmonary fibrosis, and even irreversible lung injury. However, many patients with early-stage ABPA have atypical symptoms, which could delay the diagnosis and lead to advanced disease at the time of diagnosis. The mean diagnostic delay was as long as 10 years in patients with ABPA who were eventually diagnosed correctly at later stages. The early diagnosis and treatment of ABPA are therefore required to prevent long-term lung tissue damage.

The unsatisfactory management of ABPA is mainly caused by a poor understanding of the disease, and the lack of standardized treatment plans. ${ }^{5}$ Although many cases of ABPA have been reported, there has been no large sample size study to comprehensively describe the clinical features, diagnosis, and prognosis in Chinese patients. In the present study, we analyzed 232 patients diagnosed with ABPA who suffered long-term disease. We retrospectively analyzed the clinical information for these patients in terms of their clinical presentation, and laboratory and radiological evaluations. The results of this study will improve our understanding of ABPA, and thus, facilitate its diagnosis and treatment.

\section{Materials and Methods Study Design}

This was a single-center retrospective study using data derived from the clinical information system for patients in a respiratory and critical care medicine department. The data of all patients diagnosed with ABPA were between January 2012 and December 2019. This study was approved by the Ethics Committee of Zhongshan Hospital of Fudan University, and was conducted in accordance with the Declaration of Helsinki.

\section{Patients}

Inclusion criteria included patients of both sexes who were adults (18 years of age or older) and whose diagnosis of ABPA was based on the criteria of the International Society for Human and Animal Mycology (ISHAM) working group or the Expert Consensus on Allergic Bronchopulmonary Aspergillosis developed by the Asthma Group of the Respiratory Division of the
Chinese Medical College of China in 2017. ${ }^{3,4}$ The exclusion criteria were not meeting the inclusion criteria mentioned above.

\section{Clinical Data Collection}

The clinical details of all patients were collected from their medical records, including demographic data, pulmonary comorbidities, clinical symptoms and laboratory examinations, thoracic computed tomography (CT) findings, diagnosis, treatment courses, and prognostic status.

The primary outcome is to analyze misdiagnosed cases of ABPA, looking for clinical features and reasons for misdiagnosis. The second outcome is to analyze the influence of different imaging characteristics and treatment regimens of ABPA on its prognosis.

\section{Serum Total IgE and Fungus-Specific lgE Levels}

Total IgE levels were assessed by quantitative enzymelinked immunosorbent assay (ELISA) (Demeditec Diagnostics GmbH, Kiel, Germany). A. fumigatus-specific IgE levels (sp-IgE) were assayed using a fluorescent enzyme immunoassay assay (UniCap Systems; Phadia, Stockholm, Sweden) according to the following criteria: grade 1-2 = sp$\operatorname{IgE}>0.35-<3.5 \mathrm{kUA} / \mathrm{L}$, grade $3-4=\mathrm{sp}-\mathrm{IgE}>3.5-<50 \mathrm{kUA} /$ $\mathrm{L}$, and grade $5-6=>50 \mathrm{kUA} / \mathrm{L}$.

\section{Chest CT}

Thoracic CT examinations were performed using a 256detector row spiral CT scanner (Philips, iCT, Holland) or a 64-detector row spiral CT scanner (GE Medical Systems, Milwaukee, WI, USA). Image data are evaluated independently by two experienced radiologists who maintain confidentiality regarding the identity and clinical characteristics of the patient. Both radiologists read the images given, and if the two radiologists disagree on the quality of the images, they discuss them together to reach a consensus score.

\section{Pulmonary Function}

Lung function was measured using a dry rolling seal spirometer (model 2130, SensorMedics, Yorba Linda, CA, USA) according to the American Thoracic Society/European Respiratory Society criteria for standardization. ${ }^{6}$ Age, sex, height and spirometry data were recorded for all patients. 


\section{Treatment}

Patients were treated with glucocorticoids with or without an antifungal agent (itraconazole/voriconazole). The initial dose of prednisone was $20-40 \mathrm{mg}$, which was then gradually decreased according to the clinician's judgment, with a full course of treatment lasting 12-14 months. Antifungal treatment with itraconazole or voriconazole $200 \mathrm{mg}$ was administered for at least 3 months. Follow-up data were collected for 2 years, and acute exacerbations of ABPA in patients receiving glucocorticoids with and without antifungal therapy were analyzed.

\section{Statistical Analysis}

Statistical analyses were carried out using SPSS (version 16.0; SPSS, Chicago, IL, USA) and graphs were drawn using GraphPad Prism (version 5; GraphPad Software, San Diego, CA, USA). The data were presented as number (percentage) or median (interquartile range), as required. Differences between continuous variables were analyzed using the Kruskal-Wallis test, and categorical variables were compared using the $\chi^{2}$ test. A P-value $\leq 0.05$ was considered significant.

\section{Results}

\section{Baseline Demographic and Clinical Characteristics}

A flowchart for the study participants is shown in Figure 1. A total of 232 patients were included in the study (112 (48.72\%) male, 120 (51.7\%) female; median age $41.81 \pm$ 18.31 years). The baseline clinical characteristics of the patients are shown in Table 1. Cough (232/232), expectoration (201/232), and wheezing (150/232) were the most common symptoms. A total of 180 (77.6\%) patients had comorbid asthma, and all patients had elevated specific A. fumigatus IgE levels.

\section{Comparison of Clinical Characteristics and Prognosis Between Subgroups Misdiagnosis of ABPA}

Of the 232 patients, 132 patients $(56.9 \%)$ had a history of misdiagnosis (Table 2). The top three misdiagnoses were bronchiectasis, bacterial pneumonia, and tuberculosis in 54 (40.91\%), $34(25.76 \%)$, and 19 (14.39\%) cases, respectively. Only $100(43.1 \%)$ patients were diagnosed correctly without delay. There were no significant differences in pulmonary function test parameters, age, or total $\operatorname{IgE}$
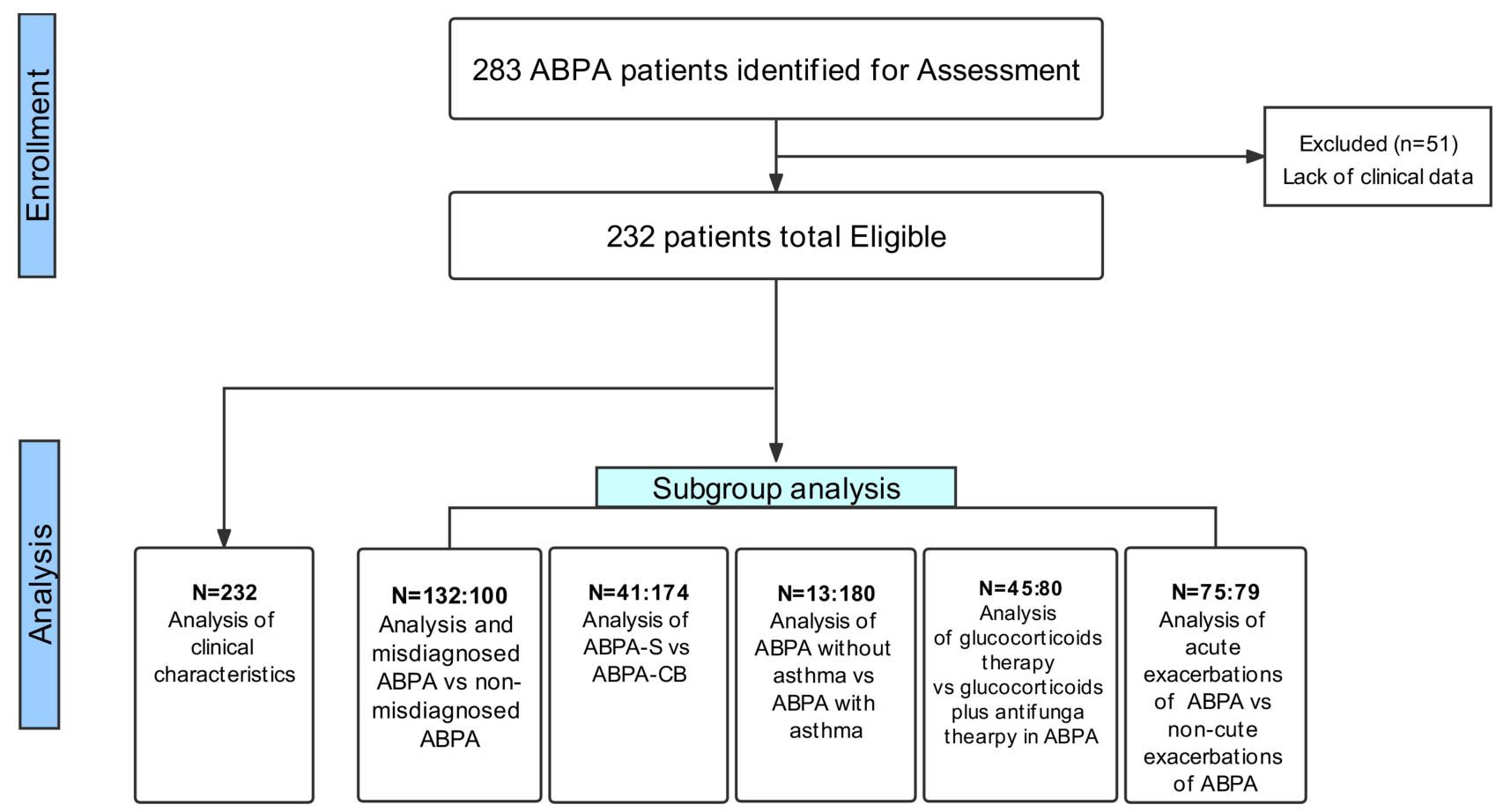

Figure I Flow diagram of study participants. 
Table I Baseline Characteristics of the Study Population

\begin{tabular}{|l|c|c|}
\hline Characteristics & $\begin{array}{c}\text { No. of } \\
\text { Patients (n) }\end{array}$ & $\begin{array}{c}\text { Percentage } \\
\text { (\%) }\end{array}$ \\
\hline Male & 112 & 48.27 \\
Smoking & 53 & 23.27 \\
History of misdiagnosis & 132 & 56.91 \\
\hline Sympotoms/signs & & \\
\hline Cough & 232 & 100.00 \\
Sputum & 201 & 86.64 \\
Wheezing & 150 & 64.66 \\
Haemoptysis & 18 & 7.76 \\
Chest pain & 18 & 7.76 \\
Fever & 97 & 41.81 \\
Night sweats & 25 & 10.76 \\
Coexisting Asthma & 180 & 77.61 \\
Allergic rhinitis & 45 & 19.40 \\
\hline Specifitic IgE test & & \\
\hline A. fumigatus & $232 / 232$ & 100.00 \\
A. fumigatus + Penicillium & $133 / 232$ & 57.33 \\
A. fumigatus + Alternaria & $88 / 232$ & 37.93 \\
Mixed mold & $47 / 232$ & 20.26 \\
\hline Laboratory examinations & & \\
\hline Total eosinophil count cell (×I0^9/ & $0.63 \pm 0.12$ & \\
L) (mean \pm SD) & & \\
Total lgE level (IU/mL) & 4173.52 & \\
& & \\
\hline
\end{tabular}

Abbreviations: lgE, immunoglobulin E; SD, standard deviation.

Table 2 Misdiagnosis of the 232 ABPA Patients

\begin{tabular}{|l|c|c|}
\hline Misdiagnosis Disease & No. of Patients (n) & Percentage (\%) \\
\hline Bronchiectasis & 54 & 40.91 \\
\hline Pneumonia & 36 & 27.27 \\
\hline Tuberculosis & 19 & 14.39 \\
\hline Bronchitis & 12 & 9.09 \\
\hline Lung cancer & 8 & 6.06 \\
\hline Lung abscess & 3 & 2.27 \\
\hline
\end{tabular}

levels between the two groups. However, nonmisdiagnosed patients were more likely to have higher total eosinophil counts, bronchiectasis, and mucus plugs revealed by $\mathrm{CT}$ (Table 3 ).

\section{ABPA Serology (ABPA-S) and ABPA Central Bronchiectasis (ABPA-CB)}

ABPA has been classified as ABPA-S and ABPA-CB, depending on the absence or presence of central bronchiectasis, respectively. Of the 232 ABPA patients, 215 had follow-up imaging, including 41 ABPA-S and 174 ABPA-CB patients. The clinical, serological, and pulmonary function data are shown in Table 4. Compared with ABPA-S, ABPA-CB was more likely to occur in older patients and in patients with high blood eosinophil levels, and poor lung function (forced vital capacity (FVC) and diffusing capacity for carbon monoxide (DLCO)). Thirty patients in the ABPA-S group and 124 in the ABPA-CB group had perfect follow-up data for 2 years. Among these, 8 patients in the ABPA-S group (26.7\%) and 67 in the ABPA-CB group (54.0\%) had recurrences ( $\mathrm{P}=0.007)$ (Table 4).

\section{ABPA with and without Asthma}

Since this is a retrospective observational study, of the 232 ABPA patients in our study, 193 ABPA patients could be determined to have asthma or not. The data of the remaining 39 ABPA patients are insufficiently accurate to determine if they have asthma. Therefore, the total number of patients in this subgroup analysis was 193. Of the 193 ABPA patients in the current study, 93\% (180/193) patients had a history of asthma. Of all 13 ABPA patients without asthma, all were diagnosed with bronchiectasis, 3 patients had also been diagnosed with COPD. Patients with a history of asthma had higher blood eosinophil counts, bronchiectasis, and poor lung function (FVC, forced expiratory volume in $1 \mathrm{~s}$ (FEV1) and FEV1/FVC). However, there was no significant difference between the two groups in sp-IgE levels and lung CT results. Furthermore, only 13 patients with follow-up data past 2 years had ABPA without asthma compared with 141 with ABPA plus asthma. Our study showed that ABPA patients with a history of asthma were more likely to relapse than ABPA patients without a history of asthma (Table 5).

\section{Corticosteroids with and without Antifungal Therapy} Most patients in this study received oral corticosteroids alone or oral corticosteroids plus antifungal therapy (itraconazole/voriconazole). A total of 125 patients with complete treatment data were divided into two groups, according to whether or not they received antifungal therapy. The clinical, serological, and spirometric data for both groups are shown in Table 6 . There were no differences in age, sex, total eosinophil count, or high-resolution CT (HRCT) findings between the two groups; however, the mean time to first ABPA exacerbation was significantly shorter in patients who received oral corticosteroids alone, 
Table 3 Comparison of Participant Characteristics Between Misdiagnosed and Correct Diagnosis Groups

\begin{tabular}{|l|c|c|c|}
\hline & Misdiagnosed (n=132) & Non-Misdiagnosed (n=100) & P value \\
\hline Age (mean \pm SD) & $49.16 \pm 12.34$ & $50.30 \pm 14.34$ & 0.77 \\
\hline Male, $n$ (\%) & $63(47.7 \%)$ & $48(48.0)$ & 0.54 \\
Coexisting Asthma, $n$ (\%) & $126(95.5 \%)$ & $93(93.0 \%)$ & 0.78 \\
\hline Laboratory examination & & & 0.001 \\
\hline Total eosinophil count cell (×I0^9/L) & $0.89 \pm 0.23$ & $1.34 \pm 0.21$ & 0.54 \\
Total IgE level (IU/mL) & $4267.12 \pm 1546.43$ & $4354.34 \pm 1276.63$ & 0.001 \\
Central bronchiectasis, n (\%) & $78(59.1 \%)$ & $96(96.0 \%)$ & 0.001 \\
Mucus plugs, $n$ (\%) & $18(13.6 \%)$ & $27(27.0 \%)$ & \\
\hline Spirometry (mean \pm SD) & & & 0.34 \\
\hline FEVI\% & $63.23 \pm 10.11$ & $66.51 \pm 12.36$ & 0.63 \\
FVC\% & $72.82 \pm 9.91$ & $74.23 \pm 11.24$ & 0.65 \\
FEVI/FVC & $63.25 \pm 8.78$ & $64.53 \pm 11.46$ & 0.43 \\
DLCO\% & $82.32 \pm 9.82$ & $83.64 \pm 9.74$ & \\
\hline
\end{tabular}

Abbreviations: IgE, immunoglobulin E; FEVI, forced expiratory volume in Is; FVC, forced vital capacity; DLCO, diffusing capacity of the lungs for carbon monoxide; SD, standard deviation.

Table 4 Comparison of Participants Characteristics Between ABPA-S and ABPA-CB

\begin{tabular}{|c|c|c|c|}
\hline & ABPA-S $(n=4 I)$ & ABPA-CB $(n=174)$ & $P$ value \\
\hline Age $($ mean $\pm S D) 2$ & $38.31 \pm 13.44$ & $42.53 \pm 13.24$ & 0.002 \\
\hline Male, n (\%) & $17(41.5 \%)$ & $80(46.0 \%)$ & 0.56 \\
\hline Mucus plugs, n (\%) & $2(4.9 \%)$ & $50(28.7 \%)$ & 0.001 \\
\hline \multicolumn{4}{|l|}{ Laboratory examination } \\
\hline Total eosinophil count cell $\left(\times 10^{\wedge} 9 / \mathrm{L}\right)($ mean $\pm S D)$ & $0.98 \pm 0.34$ & $1.85 \pm 0.65$ & 0.001 \\
\hline Total lgE level $(\mathrm{IU} / \mathrm{mL})($ mean $\pm \mathrm{SD})$ & $4 \mid 46.34 \pm 1723.43$ & $4572.32 \pm 1366.76$ & 0.04 \\
\hline \multicolumn{4}{|l|}{ Sp-lgE A. fumigatus } \\
\hline Grade I-2 & $14(34.1 \%)$ & $29(26.7 \%)$ & \\
\hline Grade 3-4 & $20(48.8 \%)$ & $116(66.7 \%)$ & \\
\hline Grade 5-6 & $7(17.1 \%)$ & $29(16.7 \%)$ & \\
\hline \multicolumn{4}{|l|}{ Spirometry (mean $\pm S D)$} \\
\hline FVC\% & $80.4 \pm 10.3$ & $78.3 \pm 9.8$ & 0.03 \\
\hline FEVI\% & $65.9 \pm 14.2$ & $62.3 \pm 10.4$ & 0.12 \\
\hline FEVI/FVC & $64.2 \pm 12.3$ & $63.7 \pm 14.3$ & 0.67 \\
\hline DLCO\% & $90.4 \pm 9.2$ & $82.6 \pm 14.5$ & 0.005 \\
\hline \multicolumn{4}{|l|}{ Clinical outcomes $(n=154)$} \\
\hline No. of 2-year follow-up & 30 & 124 & \\
\hline No. of relapses, n (\%) & $8(26.7 \%)$ & 67 (54.0\%) & 0.007 \\
\hline
\end{tabular}

Abbreviations: ABPA-S, ABPA-serum; ABPA-CB, ABPA-central bronchiectasis; Sp-IgE, Aspergillus-specific immunoglobulin E; FEVI, forced expiratory volume in Is; FVC, forced vital capacity; DLCO, diffusing capacity of the lungs for carbon monoxide; SD, standard deviation.

compared with those treated with oral corticosteroids plus antifungal treatment $(13.91 \pm 3.13$ months vs $15.6 \pm 2.12$ months, $\mathrm{P}=0.02$ ). This indicated that the addition of an antifungal agent could prolong the time to the first acute exacerbation and reduce the risk of acute exacerbation (Table 6). 
Table 5 Comparison of Participants Characteristics Between with or without Asthma

\begin{tabular}{|c|c|c|c|}
\hline & $\begin{array}{l}\text { ABPA without Asthma } \\
\qquad(n=13)\end{array}$ & $\begin{array}{l}\text { ABPA with Asthma } \\
\qquad(n=180)\end{array}$ & $P$ value \\
\hline $\begin{array}{l}\text { Age (mean } \pm \text { SD) } \\
\text { Male, } n(\%)\end{array}$ & $\begin{array}{l}49.0 \pm 16.8 \\
6(46.2 \%)\end{array}$ & $\begin{array}{l}52.5 \pm 14.4 \\
85(47.2 \%)\end{array}$ & $\begin{array}{l}0.12 \\
0.26\end{array}$ \\
\hline \multicolumn{4}{|l|}{ Laboratory examination } \\
\hline $\begin{array}{l}\text { Total eosinophil count cell }\left(\times 10^{\wedge} 9 / \mathrm{L}\right)(\text { mean } \pm \mathrm{SD}) \\
\text { Total IgE level }(\mathrm{IU} / \mathrm{mL})(\text { mean } \pm \mathrm{SD}) \\
\text { Sp-lgE A. fumigatus } \\
\text { Grade } 1-2 \\
\text { Grade } 3-4 \\
\text { Grade } 5-6\end{array}$ & $\begin{array}{c}1.54 \pm 0.32 \\
2814 \pm 1795 \\
4(30.8 \%) \\
6(46.1 \%)_{-} \\
3(23.1 \%)\end{array}$ & $\begin{array}{c}1.76 \pm 0.34 \\
3527 \pm 2730 \\
42(23.3 \%) \\
106(58.9 \%) \\
32(17.8 \%)\end{array}$ & $\begin{array}{c}0.001 \\
0.22\end{array}$ \\
\hline \multicolumn{4}{|l|}{ HRCT findings } \\
\hline $\begin{array}{l}\text { Central Bronchiectasis, n (\%) } \\
\text { Mucus plugs, n (\%) }\end{array}$ & $\begin{array}{l}13(100) \\
4(30.77)\end{array}$ & $\begin{array}{l}|6|(89.44) \\
4 \mid(22.78)\end{array}$ & $\begin{array}{l}0.02 \\
0.21\end{array}$ \\
\hline \multicolumn{4}{|l|}{ Spirometry (mean $\pm S D$ ) } \\
\hline $\begin{array}{l}\text { FVC\% } \\
\text { FEVI\% } \\
\text { FEVI/FVC }\end{array}$ & $\begin{array}{l}93.9 \pm 13.72 \\
90.0 \pm 11.19 \\
77.6 \pm 12.12\end{array}$ & $\begin{array}{l}79.4 \pm 12.82 \\
64.8 \pm 19.99 \\
66.3 \pm 16.72\end{array}$ & $\begin{array}{l}0.001 \\
0.001 \\
0.001\end{array}$ \\
\hline \multicolumn{4}{|l|}{ Clinical Outcomes $(n=154)$} \\
\hline $\begin{array}{l}\text { No. of } 2 \text {-year follow-up } \\
\text { The mean time to first ABPA exacerbation (mean } \pm S D) \text {, m }\end{array}$ & $\begin{array}{c}13 \\
15.38 \pm 3.11\end{array}$ & $\begin{array}{c}|4| \\
\mid 3.67 \pm 2.64\end{array}$ & 0.03 \\
\hline
\end{tabular}

Abbreviations: Sp-IgE, Aspergillus-specific immunoglobulin E; FEVI, forced expiratory volume in Is; FVC, forced vital capacity; DLCO, diffusing capacity of the lungs for carbon monoxide; SD, standard deviation.

\section{Longitudinal Follow-Up}

A total of 154 ABPA patients were followed-up for 2 years, and 75 patients had acute exacerbations. Univariate analysis identified older age, hemoptysis, a more prolonged course of asthma before the diagnosis of ABPA, longer duration of misdiagnosis, presence of mucus thrombus on $\mathrm{CT}$, and poorer lung function indicators as factors significantly associated with acute exacerbations of ABPA (Table 7). Older age, duration of misdiagnosis, presence of mucus thrombus on $\mathrm{CT}$, and poorer lung function remained significant prognostic indicators in multivariate analysis (Table 8).

\section{Discussion}

ABPA is a complex disorder caused by a hypersensitive immune reaction to antigens released by Aspergillus species, usually A. fumigatus. Our findings indicated that the clinical symptoms of ABPA are atypical and can often be mistaken for other conditions. Compared with ABPA-S, patients with ABPA-CB had more mucus plugs, higher total eosinophil counts and total IgE, and poorer lung function parameters (FVC, DLCO). In addition, ABPA patients with asthma had poorer lung function and were more likely to experience exacerbations within a 2-year follow-up period compared with ABPA patients without asthma. Subgroup analysis showed that ABPA patients who received glucocorticoids plus antifungal drugs had a longer time before exacerbation than ABPA patients who received glucocorticoids alone. Furthermore, univariate and multivariate analyses identified duration of asthma history, duration of misdiagnosis, mucus thrombus, and poor pulmonary function as risk factors for acute exacerbation in patients with ABPA.

Radiologically, ABPA-CB is characterized by central bronchiectasis with recurrent episodes of mucus plugging. Previous studies suggested that ABPA-S may represent the earliest stage of the disease, with less-severe immunologic findings and less propensity for relapse. ${ }^{7,8}$ In a small sample of 11 patients with ABPA-S and 20 patients with ABPA$\mathrm{CB}$, the levels of specific IgE against $A$. fumigatus and total 
Table 6 Comparison of Treatment with Glucocorticoids and Glucocorticoids Plus Antifungal Drugs

\begin{tabular}{|c|c|c|c|}
\hline & $\begin{array}{l}\text { Glucocorticoids } \\
\qquad(n=45)\end{array}$ & $\begin{array}{l}\text { Glucocorticoids Plus Antifungal Drugs } \\
\qquad(\mathrm{n}=80)\end{array}$ & $P$ value \\
\hline Age (mean $\pm S D)$ & $51.73 \pm 14.42$ & $54.81 \pm 14.54$ & 0.56 \\
\hline Male, n (\%) & $26(57.8 \%)$ & $42(52.5 \%)$ & 0.43 \\
\hline Mucus plugs, n (\%) & $4(8.89)$ & $7(8.75)$ & 0.81 \\
\hline $\begin{array}{l}\text { Total eosinophil count cell }\left(\times 10^{\wedge} 9 / \mathrm{L}\right) \text { (mean } \pm \\
\text { SD) }\end{array}$ & $1.49 \pm 0.55$ & $1.54 \pm 0.88$ & 0.84 \\
\hline Total IgE level $(\mathrm{IU} / \mathrm{mL})($ mean \pm SD) & $4393.56 \pm 1631.37$ & $4447.42 \pm 1456.34$ & 0.51 \\
\hline \multicolumn{4}{|l|}{ HRCT findings } \\
\hline Central Bronchiectasis, n (\%) & 38 (84.4\%) & 67 (83.8\%) & 0.99 \\
\hline Mucus plugs, n (\%) & 17 (37.8\%) & 27 (33.8\%) & 0.75 \\
\hline \multicolumn{4}{|l|}{ Spirometry (mean \pm SD) } \\
\hline $\mathrm{FVC} \%$ & $80.82 \pm 10.81$ & $78.34 \pm 15.86$ & 0.76 \\
\hline FEVI\% & $68.14 \pm 15.87$ & $67.13 \pm 13.86$ & 0.84 \\
\hline FEVI/FVC & $70.11 \pm 12.37$ & $67.12 \pm 14.58$ & 0.43 \\
\hline DLCO\% & $83.44 \pm 11.25$ & $82.65 \pm 14.57$ & 0.75 \\
\hline \multicolumn{4}{|l|}{ Clinical outcomes (mean \pm SD) } \\
\hline $\begin{array}{l}\text { The mean time to first ABPA exacerbation, } \\
\text { month }\end{array}$ & $13.9 \mid \pm 3.13$ & $15.64 \pm 2.12$ & 0.02 \\
\hline
\end{tabular}

Abbreviations: IgE, immunoglobulin E; FEVI, forced expiratory volume in Is; FVC, forced vital capacity; DLCO, diffusing capacity of the lungs for carbon monoxide; SD, standard deviation.

Table 7 Univariate Analysis of the Factors Associated with Acute ABPA Exacerbation for I54 Patients After 2-Year Follow-UP

\begin{tabular}{|c|c|c|c|}
\hline & $\begin{array}{l}\text { Acute Exacerbation of ABPA } \\
\qquad(n=75)\end{array}$ & $\begin{array}{l}\text { Non-Acute Exacerbation of ABPA } \\
\qquad(n=79)\end{array}$ & $P$ value \\
\hline Age (mean $\pm S D)$ & $53.42 \pm 15.78$ & $45.12 \pm 13.64$ & 0.01 \\
\hline Male, n (\%) & $42(56 \%)$ & $37(46.8 \%)$ & 0.49 \\
\hline Haemoptysis, n (\%) & $10(13.33)$ & $6(7.59)$ & 0.02 \\
\hline Mucus plugs, n (\%) & $33(44.00)$ & $20(25.31)$ & 0.01 \\
\hline Coexisting Asthma, n (\%) & $70(93.33)$ & 71 (89.87) & 0.2 \\
\hline Duration of asthma (year) (mean $\pm S D$ ) & $7.31 \pm 3.32$ & $5.53 \pm 2.31$ & 0.01 \\
\hline $\begin{array}{l}\text { Duration of misdiagnosis (year) (mean } \\
\pm S D \text { ) }\end{array}$ & $5.81 \pm 1.33$ & $4.21 \pm 1.41$ & 0.01 \\
\hline $\begin{array}{l}\text { Total eosinophil count cell }\left(\times 10^{\wedge} 9 / \mathrm{L}\right) \\
(\text { mean } \pm \text { SD) }\end{array}$ & $1.87 \pm 0.54$ & $1.52 \pm 0.64$ & 0.01 \\
\hline Total IgE level (IU/mL) (mean \pm SD) & $3850.54 \pm 769.23$ & $4428.54 \pm 670.06$ & 0.5 \\
\hline \multicolumn{4}{|l|}{ HRCT findings } \\
\hline Mucus plugs, n (\%) & $37(49.33)$ & $19(24.05)$ & 0.01 \\
\hline \multicolumn{4}{|l|}{ Spirometry (mean \pm SD) } \\
\hline FEVI\% & $43.21 \pm 9.13$ & $69.51 \pm 14.74$ & 0.01 \\
\hline FVC\% & $73.93 \pm 10.12$ & $78.21 \pm 18.64$ & 0.02 \\
\hline FEVI/FVC & $57.13 \pm 14.75$ & $65.61 \pm 13.45$ & 0.01 \\
\hline DLCO\% & $81.21 \pm 8.91$ & $83.41 \pm 11.25$ & 0.05 \\
\hline
\end{tabular}

Abbreviations: IgE, immunoglobulin E; FEVI, forced expiratory volume in Is; FVC, forced vital capacity; DLCO, diffusing capacity of the lungs for carbon monoxide; SD, standard deviation. 
Table 8 Multivariate Analysis of the Factors Associated with Acute ABPA Exacerbation for I54 Patients After 2-Year Follow-UP

\begin{tabular}{|l|c|c|}
\hline & OR (95\% Cl) & P value \\
\hline Duration of asthma (year) (mean \pm SD) & $1.62(1.02-2.58)$ & 0.01 \\
Duration of misdiagnosis (year) (mean \pm SD) & $0.43(0.65-3.42)$ & 0.01 \\
\hline HRCT findings & & 0.04 \\
\hline Mucus plugs, n (\%) & $2.20(0.31-15.65)$ & \\
\hline Spirometry, (mean \pm SD) & & 0.03 \\
\hline FEVI\% & $0.52(0.21-3.04)$ & 0.04 \\
FEVI/FVC & $1.85(1.87-65.87)$ & \\
\hline
\end{tabular}

Abbreviations: FEVI, forced expiratory volume in Is; FVC, forced vital capacity; SD, standard deviation.

IgE were both significantly lower in patients with ABPA-S compared with those with ABPA-CB, and spirometric changes were also milder in patients with ABPA-S. ${ }^{9}$ In accordance with our present results, patients with ABPA-S lack demonstrable lung damage, suggesting that this represents a milder clinical form of the disease, and in a previous a study of 55 patients with ABPA-S followed-up for 6 years, no patients developed ABPA. ${ }^{10}$ However, the inflammatory factors responsible for bronchiectasis in patients with ABPA-CB remain unknown. Bronchiectasis develops at sites of previous infiltrates, and chronic colonization by A. fumigatus may lead to continuing bronchial wall damage. The host response to Aspergillus species in the bronchial mucosa is considered to be an important factor determining the development of bronchiectasis in some patients, while others develop Aspergillus-sensitive asthma or ABPA-S.

ABPA usually accompanies asthma and CF. Gernez et al showed that patients with CF-ABPA were more likely to have elevated A. fumigatus-specific IgE and were less likely to have co-infection with Staphylococcus aureus. ${ }^{11}$ However, no patients with ABPA in combination with CF were identified in our study population. Only a few $\mathrm{CF}$ patients have been described in literature in China. Guo et al collected the clinical and genetic information of 71 Chinese CF patients and found compared with Caucasians, Chinese CF patients often present atypical symptoms, mainly displaying symptoms of pulmonary infection with fewer digestive symptoms. ${ }^{12}$ An ethnicity-specific cystic fibrosis transmembrane conductance regulator (CFTR) gene variant spectrum was also observed in $\mathrm{CF}$ patients of Chinese origin, which differed from pathogenic mutation in CF patients of Caucasian origin. ${ }^{12}$ Therefore, molecular characterization of Chinese-specific CFTR mutations should be developed to help realize more precise treatment for Chinese CF patients. ${ }^{12,13}$

In the meantime, ABPA with asthma is likely to be misdiagnosed, and delayed treatment can lead to worsening lung function and bronchiectasis, as well as more severe asthma symptoms. The current study showed that patients with asthma had higher total eosinophil counts, were more prone to bronchiectasis and mucus plugs, and had poorer lung function parameters (FVC, FEV1, and FEV1/FVC) than those without asthma. Accordingly, a previous study tried to improve the diagnosis of ABPA in asthma by defining optimal cut-off values for total and A. fumigatus-specific $\mathrm{IgE}$ and $A$. fumigatus-specific IgG levels, ${ }^{14}$ or by refinement of chest HRCT imaging findings, such as the occurrence of high-attenuation mucus impaction. ${ }^{15}$ Muthu et al also showed that ABPA without asthma behaved differently from ABPA complicating asthma. ${ }^{16}$ Compared with ABPA patients with asthma, ABPA patients without asthma had fewer acute exacerbations. However, only 13 patients in the current cohort had ABPA without asthma. ABPA usually accompanies asthma and $\mathrm{CF}$, though few studies have examined ABPA in relation to other complicating diseases, thus, discouraging its further exploration.

The core principles of managing ABPA include reducing pulmonary inflammation with glucocorticoids and reducing fungal burden in the airways with antifungal triazoles. ${ }^{3}$ Since ABPA itself may often be complicated by infection or even recurrent, the current study design thus aimed to compare the effectiveness of glucocorticoids and glucocorticoids plus itraconazole/voriconazole for the treatment of ABPA. A study of 191 patients received prednisolone or prednisolone plus itraconazole therapy in acute stage ABPA (94 patients received prednisolone therapy, 97 patients received prednisolone plus 
itraconazole therapy), Agarwal et al suggested that there was a trend towards a decline in ABPA exacerbations at 1 -year with the prednisolone plus itraconazole combination than prednisolone monotherapy. ${ }^{17}$ Similar to previous studies, our results found no difference between the glucocorticoids with and without itraconazole/voriconazole groups in terms of sex, age, bronchial mucus plugs, bronchiectasis, and lung function indicators. However, the time to first exacerbation was significantly longer in patients who received glucocorticoids plus itraconazole compared with those who received glucocorticoids alone. However, an unresolved issue is that none of the above studies have detailed data to demonstrate whether cumulative systemic steroid exposure plays a confounding role in ABPA treatment.

It is worth mentioning that increasingly biological agents are being used in ABPA as alternative modes to steroids and/or antifungals. Several studies showed efficacy for anti-IgE antibody Omalizumab, ${ }^{18}$ IL5/IL5-R $\alpha$ antibodies, ${ }^{19}$ and IL4/IL13 antibodies $^{20}$ in the treatment of ABPA. There are also ABPA patients treatmenting with Omalizumab (unpublished data).

Previous studies found that ABPA patients with acute exacerbations were more likely to have recurrent transient infiltrations on chest radiographs, with significantly increased numbers of eosinophils and IgE levels. ${ }^{21}$ Agarwal et al compared ABPA patients with and without central bronchiectasis and found that mucus was an independent predictor of frequent relapses, as confirmed in a multivariate model. ${ }^{7}$ Our current result was in line with previous studies, and multivariate analysis showed that mucus plug and decreased lung function were independent factors predicting acute exacerbation of ABPA. This may be because excess mucus and airway architecture distortion allow fungal germination and protection from immune attack, with a consequent inflammatory reaction, and further support the idea that mucus plugs containing fungi may further reduce lung function and cause acute exacerbations. Regarding the relationship between predicted acute exacerbations in these studies, we speculated that ABPA is based on an immunemediated response to $A$. fumigatus antigen, in which the presence of $A$. fumigatus in the lower respiratory tract will trigger IgE-mediated hypersensitivity reactions, leading to airway inflammation, bronchospasm, and bronchiectasis. However, further studies are needed to evaluate the reliability of mucus plugs as an objective predictor of the risk of future deterioration.
However, there are some limitations to our study. ABPA is a rare disease and we wanted to include as many patients with ABPA as possible to make the data more credible, but since this was a retrospective study to investigate the clinical characteristics and real-world management of ABPA in China, the data are somewhat incomplete. Therefore, we divided the available data into different subgroups for analysis, expecting more valuable results.

\section{Conclusions}

The misdiagnosis rate of ABPA was high. ABPA patients with a history of asthma and/or central bronchiectasis on HRCT are prone to frequent acute exacerbations. Glucocorticoids combined with antifungal drugs can prolong the time to the first acute exacerbation of ABPA. Longer durations of asthma history and misdiagnosis, mucus thrombus, and poor pulmonary function are risk factors for an acute exacerbation of ABPA.

\section{Author Contributions}

All authors contributed to data analysis, drafting, or revising the article, have agreed on the journal to which the article will be submitted, gave final approval to the version to be published, and agree to be accountable for all aspects of the work.

\section{Funding}

This work was supported by the Quality control and management system for whole procedure of precision medicine (2017YFC0910003) and Integrated application for standard system of precision medicine (2017YFC0910000), Clinical Research Special Fund of Zhongshan Hospital, Fudan University (2020ZSLC26), Shanghai Shenkang Hospital Development Center Clinical Science and Technology Innovation Project (SHDC2020CR1002A).

\section{Disclosure}

The authors report no conflicts of interest in this work.

\section{References}

1. Tracy MC, Okorie CUA, Foley EA, Moss RB. Allergic bronchopulmonary aspergillosis. J Fungi. 2016;2(2). doi:10.3390/jof2020017

2. Agarwal R, Sehgal IS, Dhooria S, et al. Allergic bronchopulmonary aspergillosis. Indian J Med Res. 2020;151(6):529-549. doi:10.4103/ ijmr.IJMR 118719

3. Agarwal R, Chakrabarti A, Shah A, et al. Allergic bronchopulmonary aspergillosis: review of literature and proposal of new diagnostic and classification criteria. Clin Exp Allergy. 2013;43(8):850-873. doi:10.1111/cea.12141 
4. Shen H, Sun Y, Lin Q, et al. Expert consensus on allergic bronchopulmonary aspergillosis developed by the Asthma Group of the respiratory division of the Chinese medical college of China. Zhonghuayixuezazhi. 2017;97(34):2650-2656.

5. Agarwal R. Burden and distinctive character of allergic bronchopulmonary aspergillosis in India. Mycopathologia. 2014;178:447-456. doi:10.1007/s11046-014-9767-z

6. Lee YB, Kim YS, Lee DH, et al. Association between HOMA-IR and lung function in Korean young adults based on the Korea national health and nutrition examination survey. Sci Rep. 2017;7(1):11726. doi:10.1038/s41598-017-11337-3

7. Agarwal R, Khan A, Gupta D, Aggarwal AN, Saxena AK, Chakrabarti A. An alternate method of classifying allergic bronchopulmonary aspergillosis based on high-attenuation mucus. PLoS One. 2010;5(12):e15346. doi:10.1371/journal.pone.0015346

8. Agarwal R, Gupta D, Aggarwal AN, et al. Clinical significance of decline in serum IgE levels in allergic bronchopulmonary aspergillosis. Respir Med. 2010;104(2):204-210. doi:10.1016/j.rmed.2009.09.005

9. Kumar R, Chopra D. Evaluation of allergic bronchopulmonary aspergillosis in patients with and without central bronchiectasis. J Asthma. 2002;39(6):473-477. doi:10.1081/jas-120004905

10. Agarwal R, Garg M, Aggarwal AN, Saikia B, Gupta D, Chakrabarti A. Serologic allergic bronchopulmonary aspergillosis (ABPA-S): long-term outcomes. Respir Med. 2012;106(7):942-947. doi:10.1016/j.rmed.2012.03.001

11. Gernez Y, Waters J, Mirkovic B, et al. Blood basophil activation is a reliable biomarker of allergic bronchopulmonary aspergillosis in cystic fibrosis. Eur Respir J. 2016;47(1):177-185. doi:10.1183/ 13993003.01068-2015

12. Guo X, Liu K, Liu Y, et al. Clinical and genetic characteristics of cystic fibrosis in Chinese patients: a systemic review of reported cases. Orphanet J Rare Dis. 2018;13(1):224. doi:10.1186/s13023-018-0968-2

13. Zhang T, Tian X, Xu K-F. Cystic fibrosis: a rare disease emerging in China. Sci China Life Sci. 2020;63(7):1082-1084. doi:10.1007/ s11427-020-1620-x
14. Agarwal R, Aggarwal AN, Garg M, Saikia B, Chakrabarti A. Cutoff values of serum $\operatorname{IgE}$ (total and A. fumigatus -specific) and eosinophil count in differentiating allergic bronchopulmonary aspergillosis from asthma. Mycoses. 2014;57(11):659-663. doi:10.1111/myc. 12214

15. Asano K, Hebisawa A, Ishiguro $T$, et al. New clinical diagnostic criteria for allergic bronchopulmonary aspergillosis/mycosis and its validation. J Allergy Clin Immunol. 2021;147(4):1261-1268 e5. doi:10.1016/j.jaci.2020.08.029

16. Muthu V, Sehgal IS, Prasad KT, et al. Allergic bronchopulmonary aspergillosis (ABPA) sans asthma: a distinct subset of ABPA with a lesser risk of exacerbation. Med Mycol. 2020;58(2):260-263. doi:10.1093/mmy/myz051

17. Agarwal R, Muthu V, Sehgal IS, et al. A randomised trial of prednisolone versus prednisolone and itraconazole in acute-stage allergic bronchopulmonary aspergillosis complicating asthma. Eur Respir J. 2021. doi:10.1183/13993003.01787-2021

18. Li JX, Fan LC, Li MH, Cao WJ, Xu JF. Beneficial effects of omalizumab therapy in allergic bronchopulmonary aspergillosis: a synthesis review of published literature. Respir Med. 2017;122:33-42. doi:10.1016/j.rmed.2016.11.019

19. Soeda S, Kono Y, Tsuzuki R, et al. Allergic bronchopulmonary aspergillosis successfully treated with benralizumab. J Allergy Clin Immunol. 2019;7(5):1633-1635.

20. Mümmler C, Kemmerich B, Behr J, Kneidinger N, Milger KA-O. Differential response to biologics in a patient with severe asthma and ABPA: a role for dupilumab? Allergy Asthma Clin Immunol. 2020;16 (1):1-4. doi:10.1186/s13223-020-00454-w

21. Knutsen AP, Bush RK, Demain JG, et al. Fungi and allergic lower respiratory tract diseases. J Allergy Clin Immunol. 2012;129(2):28091;quiz 292-3. doi:10.1016/j.jaci.2011.12.970
Journal of Asthma and Allergy

\section{Publish your work in this journal}

The Journal of Asthma and Allergy is an international, peer-reviewed open-access journal publishing original research, reports, editorials and commentaries on the following topics: Asthma; Pulmonary physiology; Asthma related clinical health; Clinical immunology and the immunological basis of disease; Pharmacological interventions and new therapies. The manuscript management system is completely online and includes a very quick and fair peer-review system, which is all easy to use. Visit http://www.dovepress.com/testimonials.php to read real quotes from published authors. 